\title{
Contribución Proximal del Nervio Ulnar para la Formación del Nervio Digital Palmar Propio Ulnar del Dedo Mínimo
}

\author{
Proximal Contribution of the Ulnar Nerve in the Formation \\ of the Ulnar Proper Palmar Digital Nerve of the Little Finger
}

"Pacheco, J. P. \& ** Olave, E.

PACHECO, J. P. \& OLAVE, E. Contribución proximal del nervio ulnar para la formación del nervio digital palmar propio ulnar del dedo mínimo. Int. J. Morphol., 27(4):1169-1172, 2009.

RESUMEN: La anatomía de la mano presenta padrones generales en la distribución de los nervios responsables de la inervación motora y sensitiva. Sin embargo, se encuentran variaciones que son de interés para la anatomía quirúrgica de la región. En una serie de 20 manos disecadas, en 19 (95\%)observamos que el nervio digital palmar propio del lado ulnar del dedo mínimo correspondía a un ramo directo del nervio ulnar y en el caso restante $(5 \%)$, de un cadáver de sexo femenino, se observó un ramo de origen antebraquial que participó en la formación del nervio digital palmar propio ulnar del dedo mínimo. Este ramo se originó desde el tronco del nervio ulnar, distal al origen del ramo dorsal de este nervio y proximal al hueso pisiforme. En su trayecto pasó a través del músculo abductor del dedo mínimo, emergiendo por la cara superficial de éste a una distancia de 11,3 mm del origen del músculo en el hueso pisiforme, uniéndose al nervio digital palmar propio del dedo mínimo, distal al hueso mencionado. A pesar que la literatura muestra diversas variaciones en la formación y distribución de los nervios en la mano, la disposición descrita es inusual y no ha sido mencionada en ella.

PALABRAS CLAVE: Anatomía; Mano; Nervio ulnar; Nervio digital palmar propio ulnar del dedo mínimo.

\section{INTRODUCCIÓN}

El conocimiento de la anatomía de los nervios de la mano es fundamental para el campo de la cirugía, debido a que ésta se encuentra expuesta a diversas lesiones laborales y de la vida cotidiana, por lo que la reparación y recuperación de tales lesiones son de extrema importancia para su funcionalidad. Sobre la mano hay en la literatura descripciones de las diversas estructuras que la componen (Kaplan, 1965; Testut \& Latarjet, 1969; Zancolli, 1983; Sunderland, 1985; Williams et al., 1995), además de variados artículos sobre la misma, donde se muestran padrones generales y variaciones. Sin embargo, siempre se ejecutan trabajos y revisiones para actualizar conocimientos y la temática de la inervación es de gran interés (Wallace \& Coupland, 1975; Homma \& Sakai, 1991,1992; Hughes \& Clarke, 1995; Torrez \& Olave, 2008; Olave \& del Sol, 2008).

La inervación sensitiva de la mano esta dada por los nervios mediano, ulnar y radial. De los dos primeros, se originan los nervios digitales palmares comunes, los cuales se dividen en los nervios digitales palmares propios. El ramo superficial del nervio ulnar, otorga el nervio digital palmar común correspondiente al cuarto espacio interóseo, donde se divide en nervio digital palmar propio ulnar del dedo anular y el nervio digital palmar propio radial del dedo mínimo, además de emitir el nervio digital palmar propio ulnar de este último dedo.

La distribución sensitiva del nervio ulnar no está exenta de variaciones, particularmente en lo que se refiere a veces, al aumento de su territorio hasta inervar parte del dedo medio (Ferrari \& Gilbert, 1991). Tambiénse ha informado de ramos del nervio ulnar de origen antebraquial y que participan en la inervación de los dedos (Olave et al., 1997; Bozkurt et al., 2004).

El ramo más medial del ramo superficial del nervio ulnar que corresponde a la inervación del dedo mínimo por su lado ulnar es en general, frecuente tanto en su origen como

"Universidad Santo Tomás, Puerto Montt, Chile; Pos-graduando, Programa de Magíster en Ciencias, Mención Morfología. Universidad de La Frontera, Temuco, Chile.

*** Facultad de Medicina, Universidad de La Frontera, Temuco, Chile.

Proyecto Didufro 120631 
en su distribución, pero también puede presentar algunas variaciones, como la que encontraron Bozkurt et al.(2002) en un miembro superior izquierdo, donde el ramo aludido se originó desde el ramo dorsal del nervio ulnar en vez de provenir de su ramo superficial.

En el presente trabajo describimos una importante variación en la formación del nervio digital palmar propio del lado ulnar del dedo mínimo, señalando su origen, trayecto y participación en la composición del nervio señalado. Sabemos que su descripción es un aporte interesante al conocimiento anatómico y a la cirugía de la mano.

\section{MATERIAL Y MÉTODO}

En el presente estudio se utilizaron 20 manos fijadas en formaldehído al $10 \%$, de individuos adultos, Chilenos, de ambos sexos, en las cuales se disecó por planos: piel, aponeurosis palmar, arco palmar superficial y nervios digitales palmares comunes y/o propios. Posteriormente, se observó la región hipotenar, identificando la inervación sensitiva y motora de la zona, donde se identificó la formación del nervio digital palmar propio ulnar del dedo mínimo, considerando su origen y trayecto. Se registró con esquemas y fotografías.

\section{RESULTADOS}

En la serie de 20 manos disecadas, en 19 (95\%) observamos que el nervio digital palmar propio del lado ulnar del dedo mínimo correspondía a un ramo directo del ramo superficial del nervio ulnar, que se dirigió oblicuamente desde su origen hacia el dedo mínimo, alcanzando a éste y distribuyéndose por su lado medial. En el caso restante (5\%), mano izquierda de un cadáver de sexo femenino, se observó un ramo de origen antebraquial que participaba en la composición del nervio digital palmar propio ulnar del dedo mínimo (Figs. 1, 2 y 3).

Este ramo se originó desde el tronco del nervio ulnar, distal al origen del ramo dorsal de este nervio, a una distancia de 41,8 $\mathrm{mm}$ proximal al hueso pisiforme. En su trayecto pasó a través de las fibras del músculo abductor del dedo mínimo, emergiendo por la cara superficial de éste a una distancia de $11,3 \mathrm{~mm}$ del origen del músculo en el hueso pisiforme, para luego unirse al nervio digital palmar propio del dedo mínimo a 18,8 mm distal al hueso mencionado.

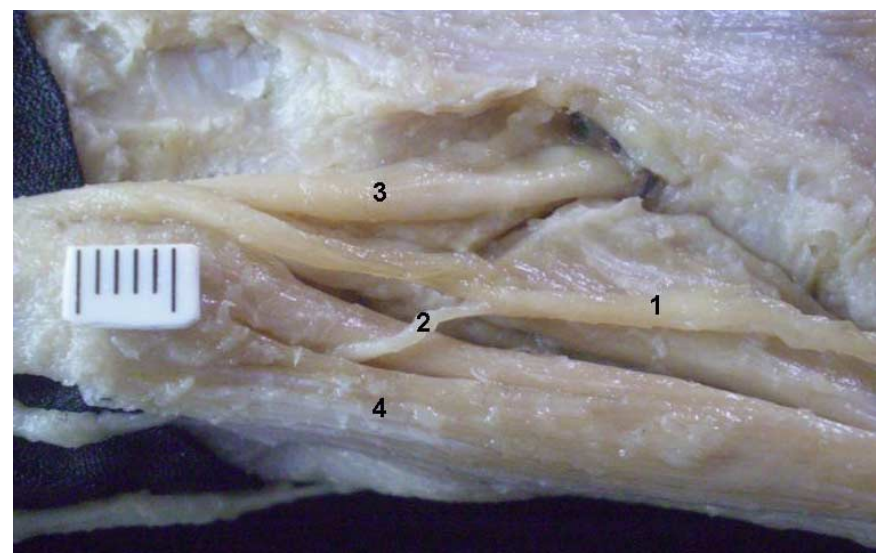

Fig. 1. 1. Constitución del nervio digital palmar propio del dedo mínimo (1) con aporte del nervio originado en la parte antebraquial del nervio ulnar (2); 3. nervio digital palmar común del $4^{\circ}$ espacio interóseo; 4. Músculo abductor del dedo mínimo.

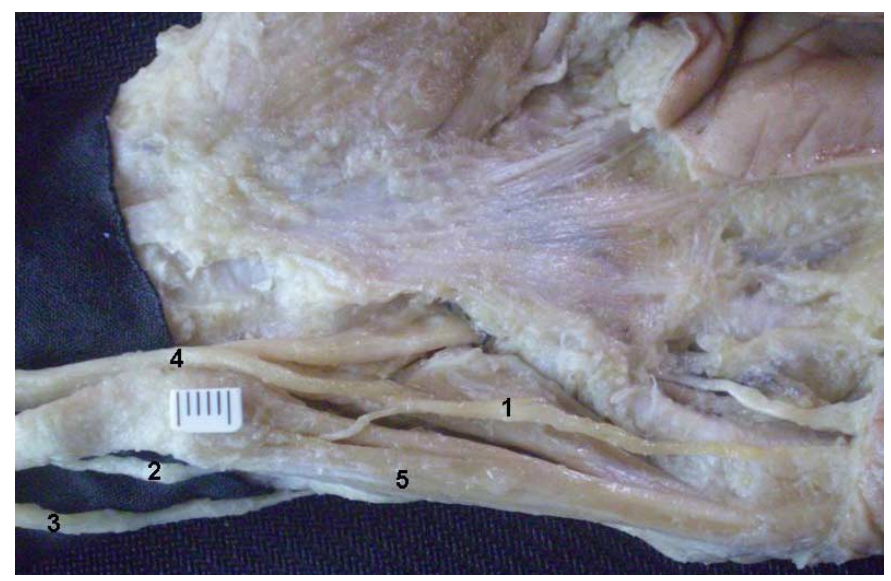

Fig. 2. Nervio digital palmar propio ulnar del dedo mínimo; 2. Ramo de origen antebraquial, uniéndose al primero; 3 . Ramo dorsal del nervio ulnar; 4. Ramo superficial del nervio ulnar; 5. Músculo abductor del dedo mínimo.

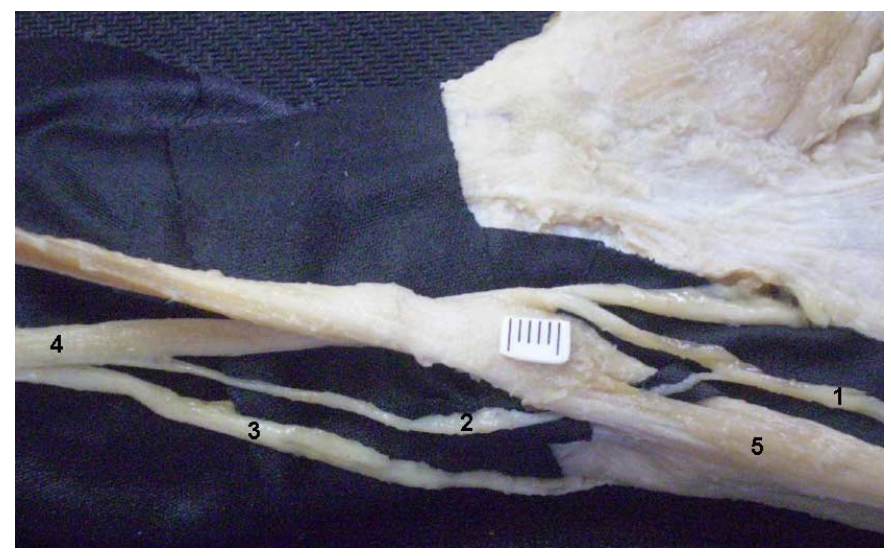

Fig. 3. Vista de la constitución del nervio digital palmar propio ulnar del dedo mínimo (1), donde se observa el origen y punto de union de éste, del ramo antebraquial (2); 3. Ramo dorsal del nervio ulnar; 4. Nervio ulnar; 5. Músculo abductor del dedo mínimo. 


\section{DISCUSIÓN}

Los nervios digitales palmares comunes y propios son derivados de los nervios mediano y ulnar, el primero de ellos dando origen a los nervios digitales palmares comunes de los espacios interóseos $1^{\circ}, 2^{\circ}$ y $3^{\circ}$, los cuales se dividen proximal a las comisuras de los dedos en nervios digitales palmares propios; el segundo, se divide en ramos superficial y profundo, a nivel de la parte media de la distancia entre los márgenes proximal y distal del retináculo de los músculos flexores, muy próximo del lado radial del pisiforme (Kaplan), siendo el primero, el responsable de emitir el nervio digital palmar común del 4o espacio interóseo y el nervio digital palmar propio del lado ulnar del dedo mínimo.

La distribución sensitiva del nervio ulnar no está exenta de variaciones, particularmente en lo que se refiere, al aumento de su territorio, llegando a inervar parte del dedo medio (Ferrari \& Gilbert).

Se han observado ramos del nervio ulnar con origen en el antebrazo, los cuales son parte de la inervación de los dedos (Olave et al.; Bozkurt et al.).

En general, la literatura clásica describe que el nervio más medial originado en el ramo superficial del nervio ulnar, corresponde a la inervación del dedo mínimo por su lado ulnar y generalmente es frecuente tanto en su origen como en su distribución (Testut \& Latarjet; Williams et al.; Moore \& Dalley). A veces se presentan variaciones, como la que mostró Bozkurt et al. en un miembro superior izquierdo, cuando el nervio mencionado se originó desde el ramo dorsal del nervio ulnar en vez de provenir de su ramo superficial.

Recientemente, McMurtrie et al. (2009) señalaron la importancia de conocer la anatomía de los nervios digitales del dedo mínimo, resaltando como la enfermedad de Dupuytren puede afectar a este dedo, sobretodo cuando se efectúa la fasciectomía palmar, ya que el nervio digital palmar propio ulnar podría ser lesionado, por lo que requiere de cuidadosa disección durante el procedimiento quirúrgico. La pérdida de sensibilidad incrementa el riesgo de lesión de este dedo.

En los casos de nuestra serie observamos que el nervio digital palmar propio ulnar del dedo mínimo se originó frecuentemente desde el ramo superficial del nervio ulnar, constatando en una de las muestras, el origen antebraquial de una porción de sus fibras, siendo una rara disposición, no encontrada en la literatura consultada, por lo tanto, de gran interés para la anatomía quirúrgica de la mano y del dedo mínimo en particular.

PACHECO, J. P. \& OLAVE, E. Proximal contribution of the ulnar nerve in the formation of the ulnar proper palmar digital nerve of the little finger. Int. J. Morphol., 27(4):1169-1172, 2009.

SUMMARY: The hand anatomy presents general patterns in motor and sensitive nerves distribution. However, is possible to find some variations that are very important for the surgical anatomy of the region. We study 20 hands of 10 cadaver individuals, fixed in $10 \%$ formaldehyde solution. We found in 19 hands (95\%) that the ulnar proper palmar digital nerve of the little finger was a direct branch of the ulnar nerve and only one case $(5 \%)$ - of female cadaver - we observed one branch originated from the forearm, which was a part in the formation of the nerve mentioned above. This branch originated from the trunk of the ulnar nerve, distal to the origin of the dorsal branch of this nerve, proximal to the pisiform bone. This nerve passed through of the abductor digiti minimi muscle and then it emerged by the superficial face of this muscle, $11,3 \mathrm{~mm}$ distal to its origin in the pisiform bone. Then, it joined to the ulnar proper palmar digital nerve of the little finger, distal to mentioned bone. Although the literature describes innumerous variations in the formation and distribution of the hand nerves, this disposition is rare and has not been mentioned.

KEY WORDS: Anatomy; Hand; Ulnar nerve; Ulnar proper palmar digital nerve.

\section{REFERENCIAS BIBLIOGRÁFICAS}

Bozkurt, M.C.; Cezayirli, E. \& Tagil, S.M. An inusual termination of the ulnar nerve in the palm. Ann. Anat., $184: 271-3,2002$.

Bozkurt, M.C.; Tagil, S.M.; Ersoy, M. \& Tekdemir, I. Muscle variations and abnormal branching and course of the ulnar nerve in the forearm and hand. Clin. Anat., 17:64$6,2004$.

Ferrari, G.P. \& Gilbert,T.A. The superficial anastomosis on the palm of the hand between the ulnar and median nerves. J. Hand Surg., 16B:511-4, 1991. 
PACHECO, J. P. \& OLAVE, E. Contribución proximal del nervio ulnar para la formación del nervio digital palmar propio ulnar del dedo mínimo. Int. J. Morphol., 27(4):1169-1172, 2009.

Homma, T. \& Sakai, T. Ramification pattern of intermetacarpal branches of the deep branch (ramus profundus) of the ulnar nerve in the human hand. Acta Anat., 141:139-44, 1991.

Homma, T. \& Sakai, T. Thenar and hipothenar muscles and their innervation by the ulnar and median nerves in the human hand. Acta Anat (Basel)., 145(1): 44-9, 1992.

Hughes ,L. A. \& Clarke, H. M. Normal arborization of the deep branch of the ulnar nerve into the interossei and lumbricals. J. Hand Surg. Am., 20(1):10-4, 1995.

Kaplan, E. B. Functional and Surgical Anatomy of the Hand. $2^{\text {nd }}$ Ed. Philadelphia, Lippincott Co., 1965.

McMurtrie, A.; Guha, A. \& Singh, R. The anatomy of the ulnar digital nerve of the little finger: a cadaveric study. J. Hand. Surg., 34E (3):397-8, 2009.

Olave, E.; del Sol, M.; Gabrielli, C; Prates, J. C. \& Rodrigues, C. F. S. The ulnar tunnel: a rare disposition of its contents. J. Anat., 191:615-6, 1997.

Olave, E. \& del Sol, M. Distribución del nervio ulnar: Innervación de los músculos interóseos, lumbricales y aductor del pulgar. Int. J. Morphol.,26(4):959-62, 2008.

Torrez, J.C. \& Olave, E. Parámetros biométricos y morfometría de la porción Terminal del nervio mediano, ramo superficial del nervio ulnar y nervios digitales palmares comunes de la mano humana. Int. J. Morphol., 26(3):675-9, 2008.

Sunderland, S. Nervios Periféricos y sus lesiones. Barcelona, Salvat, 1985.

Testut, L. \& Latarjet, A. Tratado de Anatomía Humana. Barcelona, Salvat, 1969. V. 3.

Wallace W.A. \& Coupland, R.E. Variations in the nerves of the thumb and index finger. J. Bone Joint. Surg., 57B (4):491-4, 1975.

Williams, R L.; Warwick, R.; Dyson, M. \& Bannister, L. H. Gray Anatomía. 37a Ed. Rio de Janeiro, GuanabaraKoogan, 1995. V. 2.

Zancolli, E. A. Cirurgia da Mão. 2a Ed. São Paulo, Roca, 1983.
Dirección para correspondencia:

Prof. Dr. Enrique Olave Riffo

Facultad de Medicina

Universidad de La Frontera

casilla 54-D

Temuco

CHILE

Email: eolave@ufro.cl

Recibido : 12-08-2009

Aceptado: 24-09-2009 